\title{
Research on Calculation Method of Harmonic Impedance
}

\author{
Xiaolong $\mathrm{Xu}^{1, \mathrm{a}}$ \\ ${ }^{1}$ North China Electric Power University(Baoding), Baoding 071000, China \\ a5864712@qq.com
}

Keywords: Power quality; Harmonic contributions; Harmonic impedance.

\begin{abstract}
We propose a method based on Improved Partial Least Squares Regression Algorithm for calculating Harmonic impedance. First, we analyze the volatility method and find it has a shortcoming. Then, we describe Improved Partial Least Squares Regression Algorithm. Finally, we demonstrate the correctness of the method by experimental simulation.
\end{abstract}

\section{Introduction}

With the popularity of power electronic equipment in modern society, harmonic pollution is becoming more and more serious. Harmonic pollution causes the voltage waveform at the common connection point to be distorted. Similar to the modern large industrial pollution of the earth, the grid is also experiencing treatment after Pollution. Also, harmonic impedance accurate calculation of the relationship between the governance effect.

There are two main ways to estimate harmonic impedance: "invasive" and "non-intervention". The non-intervention is more widely in the application of the project, such as analytic method, approximate calculation method, volatility method. The analytic method is used to calculate the harmonic impedance of the components of the system according to the circuit relationship. However, since the harmonic impedance model of the component is not accurate, the error of the calculation result is difficult to control. At the same time, the analytic method is computationally expensive and does not apply to the harmonic impedance calculation of large systems. The approximate calculation method is that the harmonic impedance is equal to the number of harmonic impedances and the product of the fundamental reactance. Because of the simplicity of the method, the method is used more widely. But it also brought errors. Volatility method is calculated by the harmonic voltage at the bus, which is the harmonic current fluctuation ratio calculation harmonic impedance. But it needs to meet the "double dominant conditions".

In order to solve the above problem, this paper studies a better method "Improved Partial Least Squares Regression Algorithm”. This method can improve the shortcomings of harmonic impedance calculation method and get very accurate results. Finally, the correctness of the method is verified by the simulation model and the processing of the measured data.

\section{Volatility Method}

The volatility method is to calculate the harmonic impedance by the ratio of the harmonic voltage to the harmonic current in PCC. Take the system shown in the figure 1 as an example, we assumed load A is the main non-linear load. Then we take load A as the user side and the rest of the load and power supply as the system side to estimate the harmonic impedance of this system.

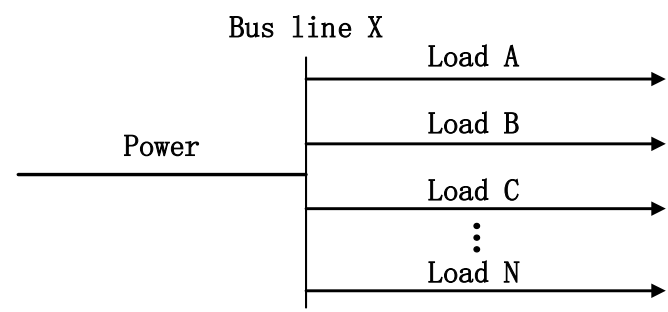

Fig. 1 The real system 
When the harmonic distortion is generated at the bus $\mathrm{X}$, the harmonic distortion can be divided into two parts produced by the user side and the system side. As shown in Figure 2, according to the Thevenin's theorem and the Norton's theorem, we will be equivalent to the system side of the voltage source and impedance in series and the user side equivalent to the current source and impedance in parallel form. Where $U_{P C C}$ is $h$ times harmonic voltage in PCC; $Z_{u}, Z_{u}$ is $h$ times the equivalent harmonic impedance in system side and user side; $U_{u}$ is the background harmonic voltage; $I_{c}$ is $\mathrm{h}$ times equivalent harmonic current source in user side.

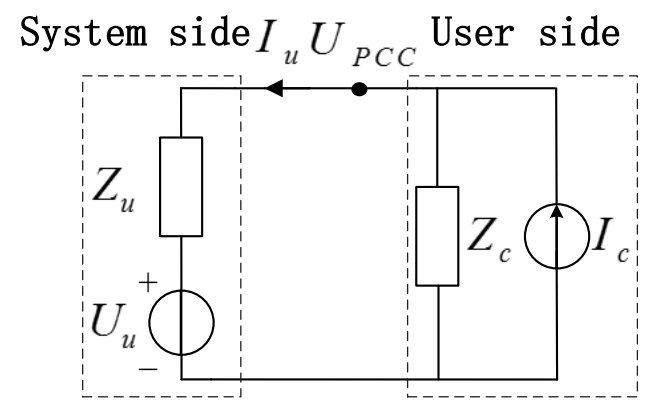

Fig. 2 System Equivalent Graph

According to the equivalent diagram, we take $h$ times harmonic as an example, and the PCC voltage can be expressed as:

$$
\dot{U}_{P C C}=Z_{u} \dot{I}_{u}+\dot{U}_{u}
$$

Measure the data of two adjacent time points, as shown in equation as follows:

$$
\begin{aligned}
& \dot{U}_{P C C 1}=Z_{u} \dot{I}_{u 1}+\dot{U}_{u 1} \\
& \dot{U}_{P C C 2}=Z_{u} \dot{I}_{u 2}+\dot{U}_{u 2}
\end{aligned}
$$

Make the above two equations subtraction:

$$
Z_{u}=\frac{\Delta \dot{U}_{P C C}}{\Delta \dot{I}_{u}}-\frac{\Delta \dot{U}_{u}}{\Delta \dot{I}_{u}}
$$

There are two assumptions about the volatility method: compared with the system side, the load side harmonics dominate; compared with the background harmonic fluctuation, the attention harmonic wave dominates. So it can be simplified as:

$$
Z_{u}=\frac{\Delta \dot{U}_{P C C}}{\Delta \dot{I}_{u}}
$$

By the above equation, the volatility method can be directly used by the adjacent time harmonic voltage fluctuation size divided by the harmonic current fluctuation size estimated harmonic impedance. Because of the convenience of the method, the method is more extensive. However, when the system does not meet the two assumptions condition of the volatility method, the error of the harmonic impedance is very large, resulting in the volatility method failure.

\section{Improved Partial Least Squares Regression Algorithm}

According to the partial least squares regression algorithm, the extraction condition of the independent variable $X$ component is meet the covariance of the largest and meet the independent set of the best integrated capacity. The composition that satisfies the extraction condition ensures that the explanatory variable is the strongest. We improved the partial least squares regression algorithm. The matrix $B$ is used to represent the space orthogonal to the dependent variable $Y$. Where the matrix $B$ consists of a feature vector $b_{1}, b_{2}, \cdots, b_{p-1}$ corresponding to its $p$ - 1 null feature root. Among them:

$$
b_{i}^{T} X^{T} Y Y^{T} X b_{i}=0
$$

Find the eigenvector of the largest eigenvalue $\lambda_{1}, \lambda_{2}, \cdots, \lambda_{s}$ of $B^{T} Y Y^{T} B$. Make $U=X B A$ It is orthogonal to the projection in direction $U$. which is: 


$$
\left(I_{n}-P_{U}\right) X=X-U\left(U^{T} U\right)^{-1} U^{T} X=X\left(I_{P}-B A\left(U^{T} U\right)^{-1} U X\right)=X D
$$

According to the return of knowledge, we can solve the errors generated by the partial least squares regression algorithm through the regression process. We will be the actual Ministry of imaginary expansion, get its regression equation:

$$
\left\{\begin{array}{l}
U_{P C C}^{\prime}=Z_{u}^{\prime} I_{u}^{\prime}-Z_{u}^{\prime \prime} I_{u}^{\prime \prime}+U_{u}^{\prime} \\
U_{P C C}^{\prime \prime}=Z_{u}^{\prime} I_{u}^{\prime \prime}-Z_{u}^{\prime \prime} I_{u}^{\prime}+U_{u}^{\prime \prime}
\end{array}\right.
$$

Where: $U_{P C C}^{\prime}, U_{P C C}^{\prime \prime}$ are the real and imaginary parts of the harmonic voltage $\dot{U}_{P C C}$ at PCC; $Z_{u}^{\prime}, Z_{u}^{\prime \prime}$ are the real and imaginary parts of the system harmonic impedance. $I_{u}^{\prime} 、 I_{u}^{\prime \prime}$ are the real and imaginary parts of the harmonic current $\dot{I}_{u}$ flowing into the system side. $U_{u}^{\prime} 、 U_{u}^{\prime \prime}$ is the real and imaginary parts of the system equivalent harmonic source $\dot{U}_{u}$.

Solve the regression equation by Improved Partial Least Squares Regression Algorithm, get the regression coefficient $Z_{u}^{\prime}$ and $Z_{u}^{\prime \prime}$. Then the system side harmonic impedance is:

$$
Z_{u}=Z_{u}^{\prime}+Z_{u}^{\prime \prime}
$$

\section{Simulation}

In order to verify the applicability of each method, build the simulation model to test it. The simulation model is shown in Figure 3. We assume $\dot{U}_{u 1}=380 \angle 30^{\circ} \mathrm{V}$ and its frequency is $50 \mathrm{~Hz}$. $\dot{U}_{u 5}=1 \angle 100^{\circ} \mathrm{V}$ and its frequency is $250 \mathrm{~Hz} . \quad \dot{I}_{c 1}=50 \angle-30^{\circ} \mathrm{A}$ and its frequency is $50 \mathrm{~Hz} . \quad \dot{I}_{c 3}=0.5 \angle 130^{\circ} \mathrm{A}$ and its frequency is $250 \mathrm{~Hz}$. In this system, the actual 5th harmonic impedance amplitude of the system is $\left|Z_{u}\right|=35.1 \Omega$, and the accuracy of each method is verified by the hypothesis parameters of the system.

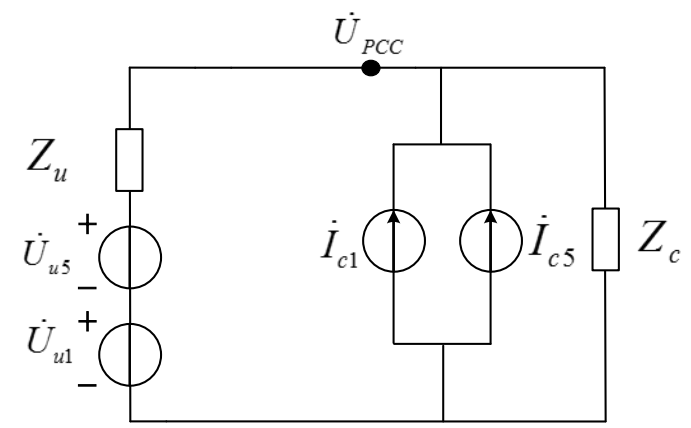

Fig. 3 The simulation of equivalent figure

We can get the calculation data from our method, and the results are shown in the figure 4 . We prove the correctness of the method.

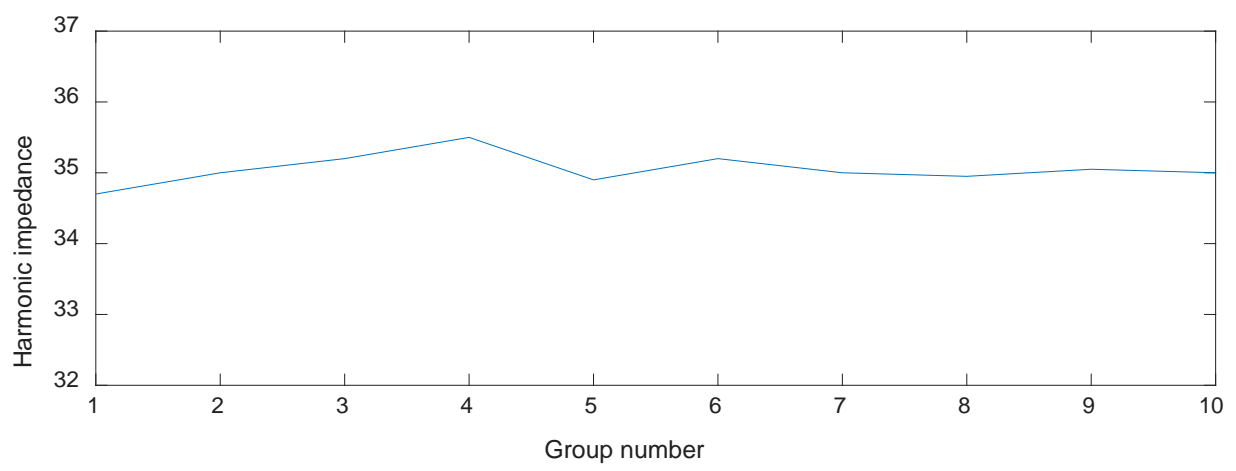

Fig. 4 The calculation results 


\section{Summary}

1) We propose a method based on Improved Partial Least Squares Regression Algorithm for Harmonic impedance calculation.

2) The correctness and validity of the method are proved by simulation experiments. So it makes sense for the actual project

\section{References}

[1] Härdle W,Liang H,Gao J T . Partially Linear Models[M] . Heidelberg:Physica Verlag,2000:19-50.

[2] Graciela Boente,Xuming He,Jianhui Zhou. Robust estimates in generalized partially linear models[J]. The Annals of Statistics,2006,34(6):2856-2878.

[3] HUI J,YANG H,LIN S. Assessing utility harmonic impedance based on the covariance characteristic of random vectors[J] . IEEE Transactions on Power Delivery,2010,25(3):1778-1786.

[4] Hooman E, Xu W. Determining the harmonic impacts of multiple harmonic-producing loads[J]. IEEE Transactions on Power Delivery, 2011, 26(2): 1187-1195.

[5] Xu W,Liu X,Liu Y. An investigation on the validity of power-direction method for harmonic source determination[J]. IEEE Transactions on Power Delivery,2003,18(1): 214-219.

[6] Li C,Xu W,Tayjasanant T. A critical impedance based method for identifying harmonic sources[J]. IEEE Transactions on Power Delivery,2004,19(2): 671-678. 